\title{
Adenoma versus Carcinoid Tumor of the Middle Ear: a Study of 48 Cases and Review of the Literature
}

\author{
Kevin R. Torske, D.D.S., M.S., Lester D.R. Thompson, M.D. \\ Department of Endocrine and Otorhinolaryngic-Head and Neck Pathology, Armed Forces Institute of \\ Pathology, Washington, D.C.
}

Carcinoid tumors and adenomas of the middle ear are rare neoplasms of indeterminate relationship to one another. Indeed, the literature is devoid of a large comprehensive series that evaluates the clinical, histologic, and immunophenotypic features of these tumors and their potential relationship. Forty-eight cases of middle ear adenoma between 1970 and 1995 were identified in the files of the Armed Forces Institute of Pathology. All cases were evaluated for cytomorphology and architectural pattern, in addition to their reactivity with various immunohistochemical reagents. Clinical follow-up was also obtained. A comprehensive review of the literature was performed with an eye toward correlating any distinct differences or similarities between carcinoid tumors and adenomas of the middle ear. The patients included 21 women and 27 men, aged 20 to 80 years (mean, 45.0 y). Patients experienced hearing loss, mass, and/or pain for a mean duration of 1.7 years. The mean tumor size was $0.8 \mathrm{~cm}$, with six tumors extending beyond the middle ear. Histologically, the tumors were moderately cellular and unencapsulated, arranged in glandular, trabecular, and solid patterns composed of small cells with "salt and pepper" nuclear chromatin distribution. The tumor cells were immunoreactive with keratin, keratin 7 , chromogranin, and human pancreatic polypeptide. All patients had surgery. No patients died with their disease (mean follow-up, 15.7 y). Eight patients developed recurrences that were treated surgically and were without evidence of disease at last follow-up (mean, $15.1 \mathrm{y})$. Our study and the review of the literature

Copyright (C) 2002 by The United States and Canadian Academy of Pathology, Inc.

VOL. 15, NO. 5, P. 543, 2002 Printed in the U.S.A.

Date of acceptance: January 30, 2002.

The opinions or assertions contained herein are the private views of the authors and are not to be construed as official or as reflecting the views of the Department of the Navy or Department of Defense.

Address reprint requests to: Lester D.R. Thompson, M.D., Department of Endocrine and Otorhinolaryngic-Head and Neck Pathology, Building 54, Room G066-11, Armed Forces Institute of Pathology, 6825 16th Street NW, Washington, DC 20306-6000; e-mail: thompsonl@afip.osd.mil; fax: 202-782-3130. showed adenomas and carcinoid tumors of the middle ear to be essentially indistinguishable benign tumors. Middle ear adenoma most correctly describes their morphologic features and clinical behavior, although neuroendocrine adenoma of the middle ear may be a more accurate designation.

KEY WORDS: Adenoma, Carcinoid, Immunohistochemistry, Middle ear, Prognosis.

\section{-}

Mod Pathol 2002;15(5):543-555

Adenomas and carcinoid tumors of the middle ear are rare neoplasms with similar, if not indistinguishable, morphologies. First described in 1976 (1, 2) and further elaborated upon in 1980 (3), these tumors have been the subject of much debate with regard to their similarities, differences, etiologies, and prognoses. Limited mostly to case reports and small series, the English-language literature is devoid of a large comprehensive evaluation of these tumors with respect to their histomorphology, immunohistochemical reactivity, treatment outcomes, and clinical behavior. We elected to undertake such a study with an eye toward identifying any distinct separation of these two tumors.

\section{MATERIALS AND METHODS}

The records of 68 patients with tumors diagnosed as "middle ear adenoma," "ceruminal gland adenoma," and "atypical middle ear adenoma" were identified in the files of the Otorhinolaryngic-Head \& Neck Registry at the Armed Forces Institute of pathology from 1970 to 1995 . No records of "carcinoid tumors" of the middle ear were located in the registry. This lack was considered not to be due to the true absence of such a tumor but more to the preferred designation of this tumor as "middle ear adenoma" by Dr. Hyams and his colleagues within the Department of Otorhinolaryngic Pathology. These 68 patients were identified in a review of 1,703 patients with primary benign or malignant neoplasms of the ear seen in consultation during this same time period. However, 20 patients were 
excluded from further consideration because of at least one of the following reasons: (1) paraffin blocks were unavailable for additional sections or immunophenotypic analysis (2); the original submitted case did not have sufficient demographic information supplied from which to obtain adequate follow-up information; and (3) the cases were diagnosed indefinitely, using terms such as consistent with, suggestive of, or suspicious for, which usually indicated a lack of sufficient diagnostic material from which to proffer a definitive diagnosis. Therefore, the remaining 48 patients with middleear adenomas $(2.8 \%$ of all ear neoplasms within the files of the AFIP) compose the subject of this study based on adequate hematoxylin and eosin-stained slides to make a definitive diagnosis and adequate demographic information for initial attempt at follow-up. Forty-three cases were obtained from civilian sources, including university medical centers and foreign contributors, four cases from military hospitals, and one case from a Veterans Administration medical center.

Materials within the Institute's files were supplemented by a review of the patient demographics (gender, age) and symptoms at presentation (mass, hearing loss, bleeding, infection, pain, nerve paralysis, discharge, equilibrium changes, tinnitus, and/or popping), including duration. In addition, we reviewed radiographic, surgical pathology, and operative reports, and obtained follow-up information from oncology data services by written questionnaires or direct communication with the treating physician(s) or the patient. Follow-up data, available for 45 patients, included information regarding tumor location, presence of recurrent or metastatic disease, treatment modalities used, and the current patient and disease status. This clinical investigation was conducted in accordance and compliance with all statutes, directives, and guidelines of the Code of Federal Regulations, Title 45, Part 46, and the Department of Defense Directive 3216.2 relating to human subjects in research.
The macroscopic pathologic observations noted within this study were garnered from the individual gross descriptions of the neoplasms by the contributing pathologists. Hematoxylin and eosin-stained slides from all patients were reviewed for morphologic assessment of middle-ear adenoma/carcinoid, as originally defined by both Hyams et al. and Murphy et al. $(2,3)$ A number of macroscopic and histologic observations were recorded for each tumor as follows: exact tumor location; tumor size (greatest dimension in centimeters); tumor encapsulation (presence or absence); tumor extension (mastoid bone, eustachian tube, and/or external auditory canal); architectural pattern of growth (solid, trabecular, organoid, infiltrating, and/or glandular); surface origin or pagetoid spread; presence or absence of necrosis; tumor cellularity (low, moderate, or high); mitotic figures (number of mitotic figures per 10 high power fields [magnification at $40 \times$ with a $10 \times$ objective lens using an Olympus BX40 microscope]); atypical mitotic figures (present or absent, and defined by abnormal chromosome spread, tripolar or quadripolar forms, circular forms, or indescribably bizarre); nuclear pleomorphism (mild, moderate or severe); presence or absence of nucleoli; and the presence of other microscopic pathologic findings.

Immunophenotypic analysis was performed in all cases with suitable material by the standardized avidin-biotin method of Hsu et al. (4) employing 4- $\mu \mathrm{m}$-thick, formalin-fixed, paraffin-embedded sections. Table 1 documents the pertinent, commercially available immunohistochemical antibody panel used. The analysis was performed on a single representative block in each primary tumor. When required, proteolytic antigen retrieval was performed by predigestion for 3 minutes with $0.05 \%$ Protease VIII (Sigma Chemical Co., St. Louis, MO) in a $0.10 \mathrm{M}$ phosphate buffer, $\mathrm{pH}$ of 7.8 , at $37^{\circ} \mathrm{C}$. Cellular conditioning to achieve antigen enhancement (recovery) was performed by using formalinfixed, paraffin-embedded tissue treated with a buff-

Table 1. Immunohistochemical Panel

\begin{tabular}{|c|c|c|c|c|}
\hline Antigen or Antibody & $\begin{array}{l}\text { Primary } \\
\text { Antibody }\end{array}$ & Company & Dilution & Antigen Recovery \\
\hline Cytokeratin & & Boehringer Mannheim & & \\
\hline AE1/AE3 & $\mathrm{mm}$ & Biochemicals, Indianapolis, IN, & $1: 50$ & Enzyme digestion \\
\hline CK1 & $\mathrm{mm}$ & and DAKO, Carpinteria, CA & $1: 200$ & \\
\hline Keratin 7 & $\mathrm{~mm}$ & DAKO & $1: 200$ & Enzyme digestion \\
\hline Keratin 20 & $\mathrm{~mm}$ & DAKO & $1: 50$ & Enzyme digestion \\
\hline CAM5.2 & $\mathrm{mm}$ & Becton Dickson, San Jose, CA & $1: 100$ & Enzyme digestion \\
\hline Chromogranin & $\mathrm{mm}$ & Boehringer Mannheim & $1: 3,200$ & None \\
\hline Synaptophysin & $\mathrm{rp}$ & DAKO & Neat & Enzyme digestion \\
\hline Serotonin & $\mathrm{mm}$ & DAKO & $1: 25$ & None \\
\hline Neuron-specific enolase & $\mathrm{rp}$ & Ventana, Tucson, AZ & Neat & None \\
\hline Human pancreatic polypeptide & $\mathrm{mm}$ & DAKO & $1: 1000$ & None \\
\hline S-100 protein & rp & DAKO & $1: 800$ & None \\
\hline Vimentin & $\mathrm{mm}$ & BioGenex Labs, San Ramon, CA & $1: 400$ & Microwave recovery \\
\hline
\end{tabular}

$\mathrm{mm}$, mouse monoclonal; rp, rabbit polyclonal. 
ered citric acid solution and heated for 20 minutes in a calibrated microwave oven. Afterwards, the sections were allowed to cool at room temperature in a citric acid buffer solution for 45 minutes before continuing the procedure. Standard positive controls were used throughout, with serum used as the negative control. The antibody reactions were graded as absent to weak $(0-1+)$, moderate $(2+-$ $3+)$, and strong $(4+)$ staining, and the fraction of positive cells was determined by separating them into four groups: $<10 \%, 11-50 \%, 51-90 \%$, and $>90 \%$.

Our review of the literature was extensive, based on a MEDLINE search from 1966 to 2001. However, for purposes of succinctness, we limited our inclusion to articles with pathology descriptions written in English.

\section{RESULTS}

\section{Clinical Demographics and Presentation}

A summary of the clinical information on the patients in this series is provided in Table 2. The patients included 21 women and 27 men, aged 20 to 80 years with a mean of 45.0 years at the time of initial clinical presentation (Table 2). The women had a slightly older mean age at presentation (47.9 y) than the men ( $42.7 \mathrm{y}$ ), but this difference was not statistically significant.

Patients presented with a variety of symptoms, but the most frequently identified single symptom was hearing loss ( $n=33$ patients; Table 2$)$. A variety of other more nonspecific symptoms were noted and included mass ( $n=12$ patients), pain $(n=11$ patients), discharge ( $n=7$ patients), equilibrium

Table 2. Clinical Features in Middle-Ear Adenomas

\begin{tabular}{lc}
\multicolumn{1}{c}{ Clinical Characteristic } & Data $(N=48)$ \\
\hline Gender, $(n)$ & 27 \\
Women & $20-80$ \\
Men & 45.0 \\
Age at presentation (y) & 47.9 \\
Range & 42.7 \\
Mean & \\
Women (mean) & 33 \\
Men (mean) & 12 \\
Symptoms at presentation ${ }^{\mathrm{a}}$ & 11 \\
Hearing loss & 16 \\
Mass & \\
Pain & \\
Bleeding, infection, discharge, nerve paralysis, & 4 \\
equilibrium changes, and/or tinnitus or & \\
popping & \\
Asymptomatic (identified incidentally during & $1-228$ \\
routine exam) & 20.8 \\
Duration of symptoms (mo) & 29.4 \\
Range & 14.1 \\
Mean & \\
Women (mean) & \\
Men (mean) & \\
\hline Patients may have presented with more than & \\
\hline
\end{tabular}

a Patients may have presented with more than one symptom; therefore the numbers do not add up to the total number of patients. changes ( $n=7$ patients), tinnitus or popping ( $n=$ 6 patients), nerve paralysis ( $n=4$ patients), infection ( $n=2$ patients), and bleeding ( $n=1$ patient). Obviously, many patients ( $n=24$ patients) presented with more than one symptom. Tumors were discovered incidentally during routine physical examination or diagnostic radiographic studies performed for nonrelated reasons in four patients.

The duration of symptoms lasted from 1 to 228 months, with a mean of 20.8 months (Table 2). Female patients had a longer average duration of symptoms (29.4 mo) than males (14.1 mo); however, this finding was skewed by two female patients with extraordinarily long symptom duration (120 and 228 mo, respectively).

\section{Radiographic Studies}

Radiographic assessments were only available for two patients. Both depicted nondestructive mass lesions within the middle ear without evidence of bone invasion. Opacification of the middle ear and mastoid air cells was noted in both patients.

\section{Pathology}

\section{Macroscopic findings}

All patients presented with unilateral disease, although more tumors presented on the right ( $n=26$ patients) than the left ( $n=22$ patients; Table 3 ). Most of the lesions were excised in a piecemeal fashion. The overall aggregate mean tumor size was $0.8 \mathrm{~cm}$, with a range of 0.2 to $3.0 \mathrm{~cm}$ (Table 3 ), with no difference in the mean tumor size between women $(0.74 \mathrm{~cm})$ versus men $(0.84 \mathrm{~cm})$ or between the right $(0.81 \mathrm{~cm})$ and left $(0.80 \mathrm{~cm})$. The tumors were noted to extend into the external auditory canal alone ( $n=2$ tumors), the external auditory canal and mastoid bone ( $n=1$ tumor), the mastoid bone alone ( $n=1$ tumor), eustachian tube and the mastoid bone $(n=1)$, and the eustachian tube alone ( $n=1$ tumor). The lesions most commonly filled the middle-ear cavity and were intimately associated with the ossicular chain.

Table 3. Macroscopic Features of Middle-Ear Adenomas

\begin{tabular}{lc}
\hline Macroscopic Feature & Data \\
\hline Primary site, $(n)$ & 26 \\
Right & 22 \\
Left & \\
Tumor size $(\mathrm{cm})$ & $0.2-3.0$ \\
Range & 0.8 \\
Mean & 3 \\
Tumor extension ${ }^{\mathrm{a}},(n)$ & 3 \\
Eustachian tube & 2 \\
Mastoid bone & \\
External auditory canal &
\end{tabular}

${ }^{a}$ Extension may have involved mastoid bone and eustachian tube or mastoid bone and external auditory canal. 
The tumors were described macroscopically as avascular, soft or rubbery unencapsulated masses with a variegated cut surface. The lesions tended to be gray-tan, brown-red, or pale yellow.

\section{Microscopic findings}

The microscopic findings are displayed in Table 4. All tumors were unencapsulated and most were of moderate cellularity. The tumors were predominantly composed of cuboidal-to-columnar cells with indistinct cytoplasmic borders (Fig. 1). Flattened, irregular, plasmacytoid, and spindled morphologies were also noted. The cytoplasm was eosinophilic and homogenous to finely granular.

The nuclei tended to be round to oval with minimal pleomorphism. Moderate to severe nuclear pleomorphism was only occasionally observed. The chromatin tended to display a "salt-and-pepper" pattern consistent with neuroendocrine origin, especially in the cells comprising the trabecular and solid patterns and those comprising the external cellular layer of the ductal structures (see below). Otherwise, the chromatin was fine and even in appearance. The nuclei were centrally or eccentrically placed, giving some cells a "plasmacytoid" appearance. Intranuclear cytoplasmic inclusions were infrequently identified. Nucleoli were inconspicuous, and mitoses were essentially absent.

Architectural patterns included glandular, trabecular, solid, organoid, and infiltrative (Fig. 2). The architecture varied between tumors and even within the same tumor (Fig. 3), with $81 \%$ (37/48) displaying more than one pattern. The glandular pattern predominated and was seen in $73 \%(35 / 48)$ of the cases, followed by trabecular $(60 \%)$, solid $(33 \%)$, infiltrative $(15 \%)$, and organoid $(8 \%)$.

The glandular pattern consisted of duct-like structures with focal "back-to-back" gland configu-

Table 4. Microscopic Features of Middle-Ear Adenomas

\begin{tabular}{lc}
\multicolumn{1}{c}{ Feature } & Data \\
\hline Growth pattern ${ }^{\text {a }}(n)$ & 35 \\
Glandular & 29 \\
Trabecular & 16 \\
Solid & 7 \\
Infiltrating & 4 \\
Organoid & 9 \\
Number with only a single pattern & 2 \\
Surface origin or pagetoid spread $(n)$ & \\
Cellularity $(n)$ & 2 \\
Low & 35 \\
Moderate & 11 \\
High & $1(<25 \%)$ \\
Necrosis $(n)$ & 3 \\
Pleomorphism, (moderate to severe), $n$ & 1 \\
Ulceration $(n)$ & 3 \\
Nucleoli $(n)$ & \\
Other histologic findings $(n)$ & 4 \\
Cholesteatoma & 2 \\
Pseudoepitheliomatous hyperplasia &
\end{tabular}

a The architecture usually included more than one pattern, except for nine cases that only presented a single histologic pattern. ration. The ducts were lined by a dual cell population composed of an inner (luminal), flattened, slightly more intensely eosinophilic cell surrounded by a basally positioned cuboidal to short columnar cell (Fig. 4). The glandular lumen was occasionally filled with an amorphous, pink substance. Papillary structures were not identified.

The trabecular pattern was composed of variably sized columnar cells with round to elongated nuclei. Clefting (considered artifactual) between the trabecular epithelial cells and the fibrous stroma was consistently identified. The solid pattern displayed variable cellular cohesiveness and also showed artifactual clefting between the solid islands and the fibrous stroma. The "infiltrative" pattern was characterized by small irregular groups and strands of cells in a moderately desmoplastic stroma. This pattern gave the illusion of the tumor cells dissecting the collagen bundles in an uncontrolled and aggressive fashion. The cells tended to be smaller than those within the other patterns with a higher nuclear-to-cytoplasmic ratio; however, other features of true aggressiveness such as mitotic activity, pleomorphism, necrosis, bone, perineural, or lymph-vascular space invasion were not noted. Finally, the organoid pattern consisted of variably sized, rounded nests of tumor cells with a polygonal or spindled morphology.

Definitive surface derivation was not seen in this clinical series. Pagetoid-like spread was noted in only two tumors. In both cases orthokeratinized squamous epithelium showed focal nests of tumor cells at all levels (Fig. 5). As the surface mucosa of the middle ear is usually a nonkeratinized, modified respiratory epithelium, the orthokeratinized squamous epithelium noted within the two cases of pagetoid spread may have indicated perforation into the external auditory canal. Reactive squamous metaplasia of the middle-ear muscosa or tympanic membrane to the presence of the underlying tumor may also explain this finding. As both biopsies were significantly fragmented, the exact location of any one tumor fragment was equivocal. One of the cases with pagetoid spread also displayed focal tumor necrosis, the only tumor within this series with such a finding. One of the patients with pagetoid spread developed recurrent disease but did not have evidence of disease at last follow-up.

\section{Immunohistochemistry}

The tumor cells were immunoreactive with a variety of keratin antibodies, including cytokeratin (CK) cocktail (89.6\%), CK7 (89.6\%), and CAM 5.2 (81.3\%; Table 5$)$. The CK reactivity was usually very intense and strong $(4+)$. CK20, when present (3 of 48 tumors), was only focally and weakly immunoreactive. 


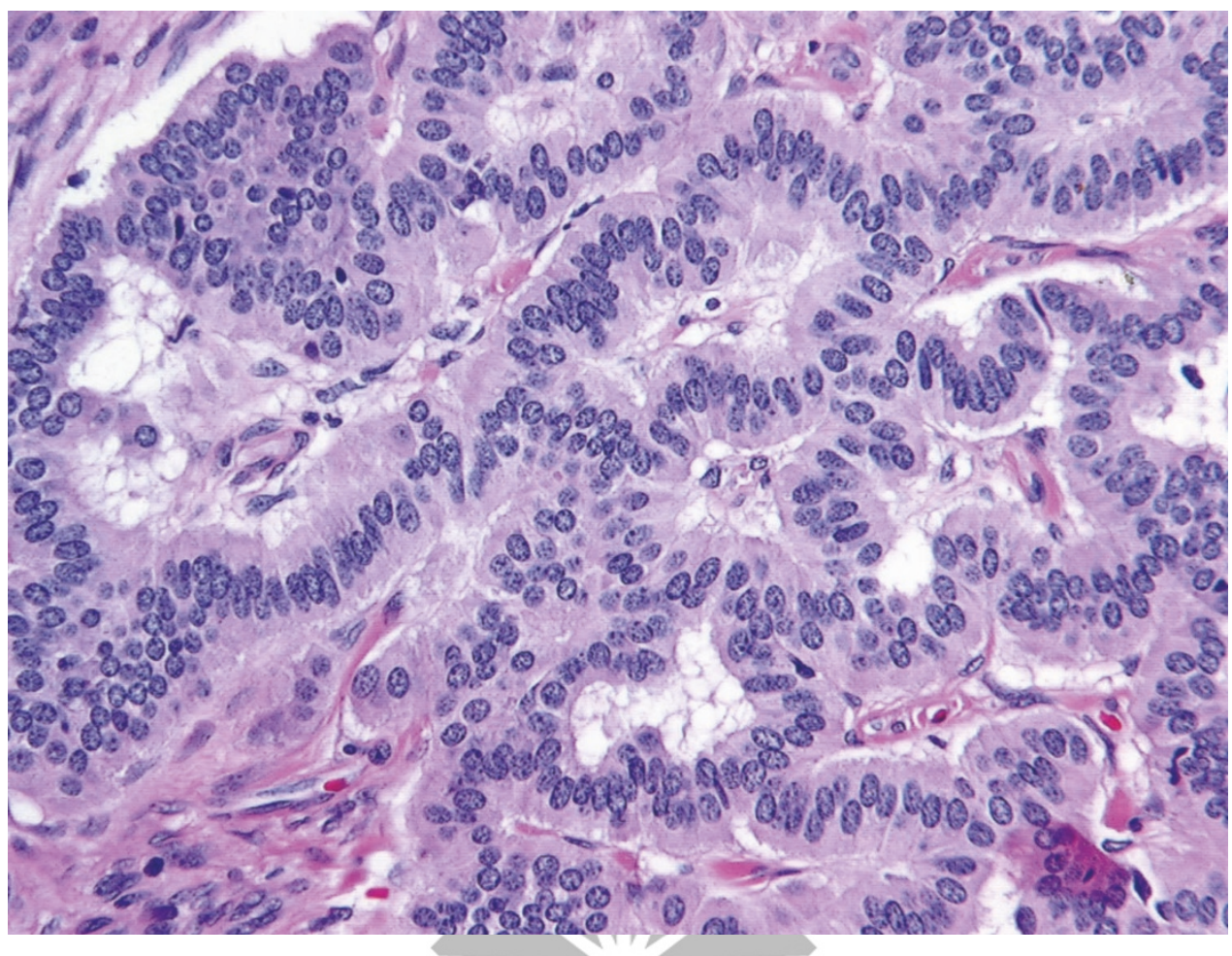

FIGURE 1. Neuroendocrine adenoma of the middle ear displaying a trabecular growth pattern composed of columnar cells with round-to-oval nuclei, 'salt-and-pepper' chromatin pattern, and eosinophilic, finely granular cytoplasm.

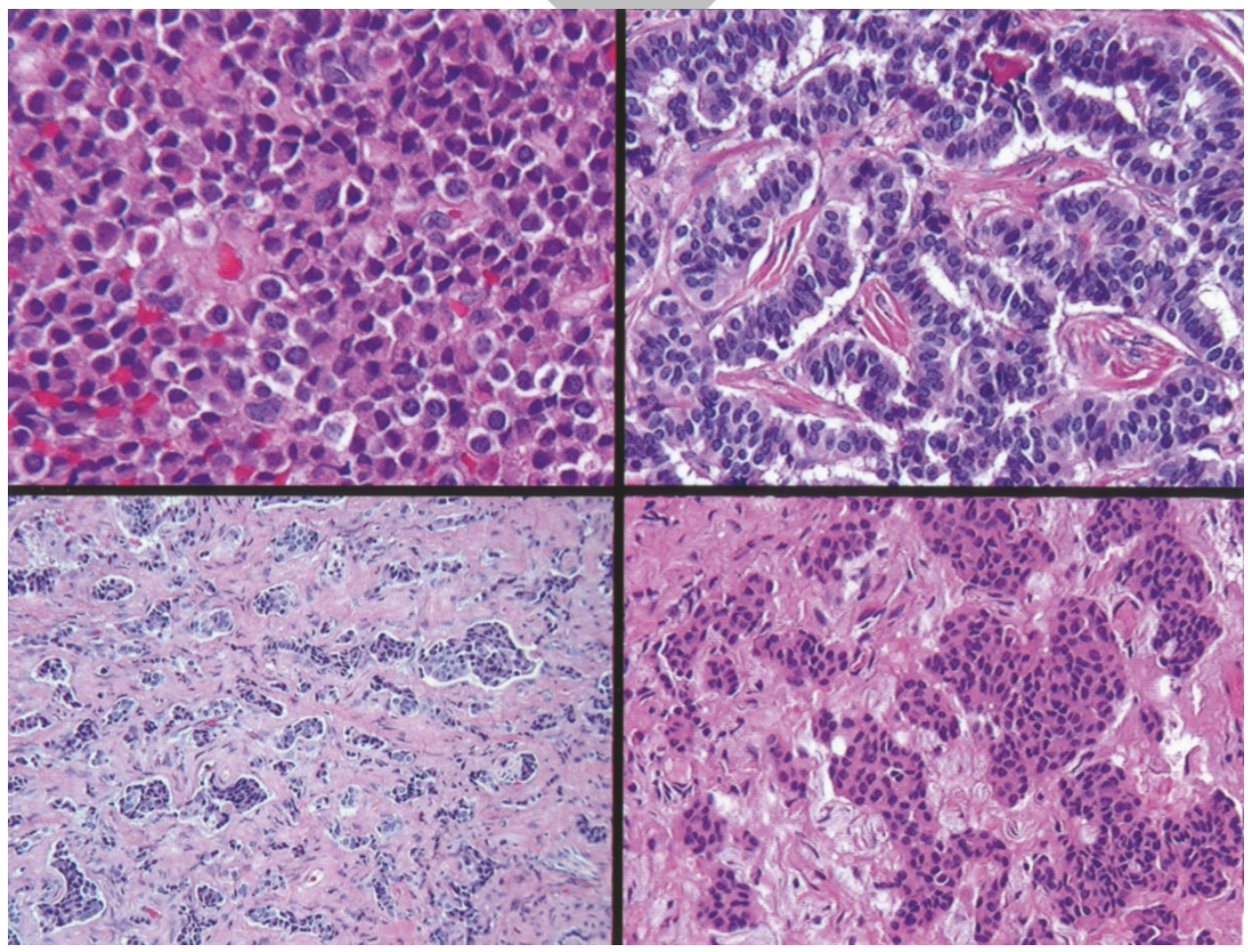

FIGURE 2. Multiple architectural patterns are present in neuroendocrine adenomas of the middle ear. Upper left, solid growth pattern with plasmacytoid cells. Upper right, trabecular growth pattern with artifactual clefting. Lower left, infiltrative pattern. Lower right, organoid pattern.

The CK immunoreactivity was primarily distributed within glandular structures. Moreover, the CK7 immunoreactivity specifically highlighted the inner (luminal) layer of the glandular cells with a uniform and intense result (Fig. 6), although it was only variably present with moderate to strong reactivity in the other architectural patterns.

Neuroendocrine marker immunoreactivity included chromogranin (87.5\%), neuron-specific enolase (NSE; 50\%), synaptophysin (31.3\%), and 


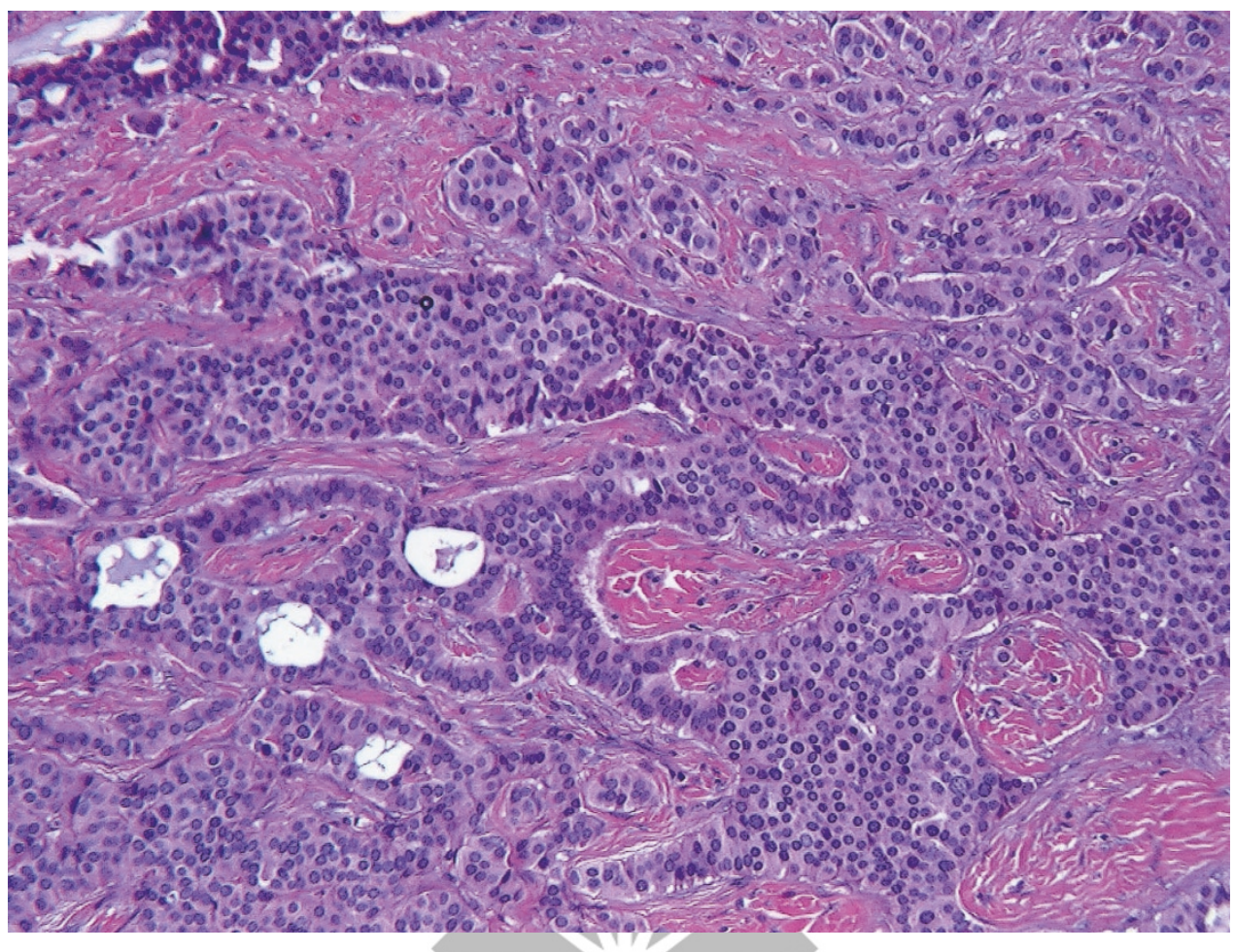

FIGURE 3. Single tumor displaying multiple growth patterns, to include infiltrative (upper right), organoid (upper central), solid, trabecular, and glandular.

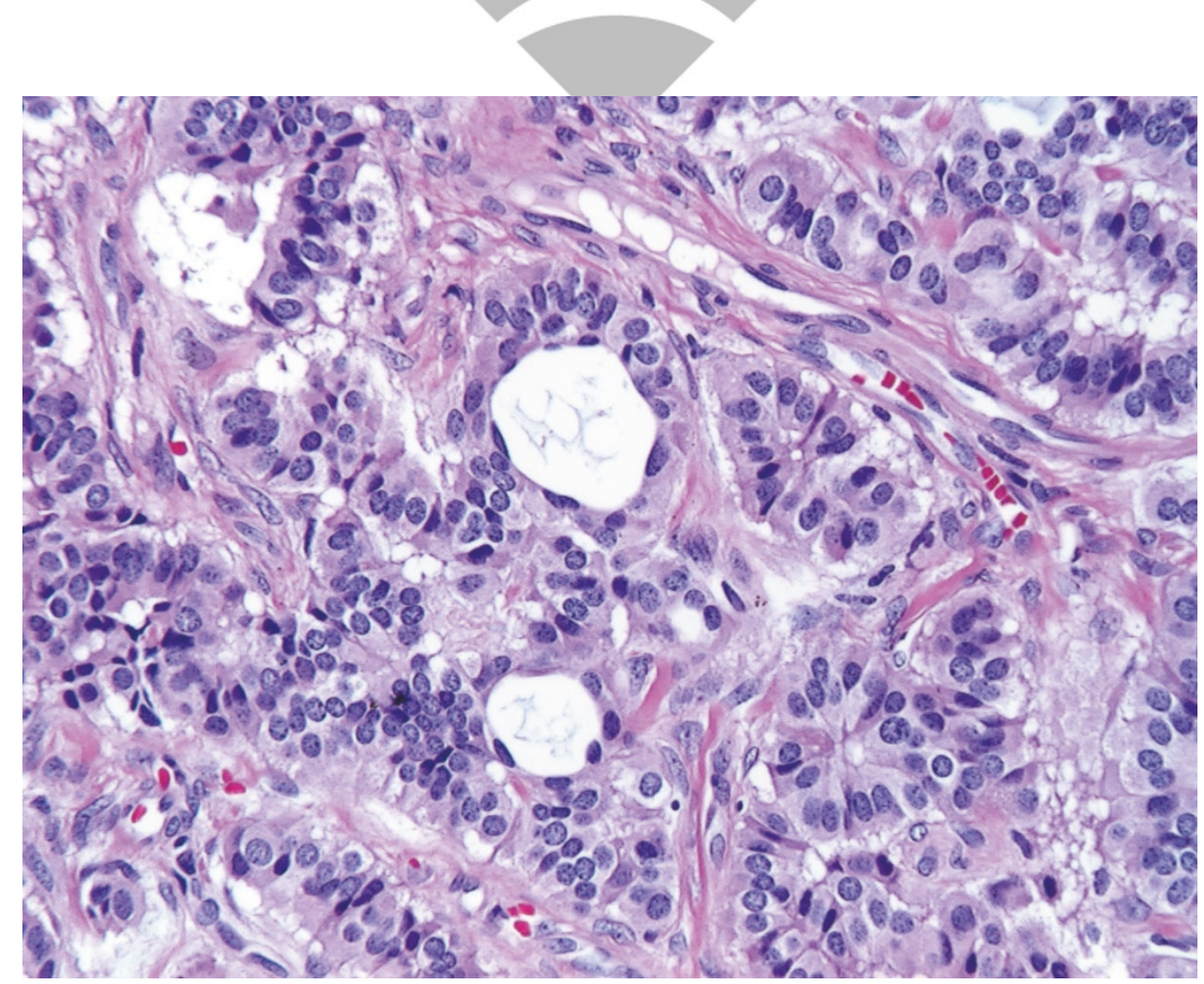

FIGURE 4. Glandular pattern with easily identifiable inner, flattened cellular layer, and an outer cuboidal-to-columnar layer. Note the lightly basophilic amorphous material present within the glandular lumina.

serotonin (25\%). Neuroendocrine markers were positive within the basal cell layer of the glandular elements while more diffusely positive in the other architectural patterns. Reactivity, however, was not uniform and varied both between cases and in level of intensity. In all but two cases, at least one neuroendocrine marker was present to a significant degree. Both of the nonreactive cases displayed a predominant glandular pattern but were positive for HPP in the basal cells. 


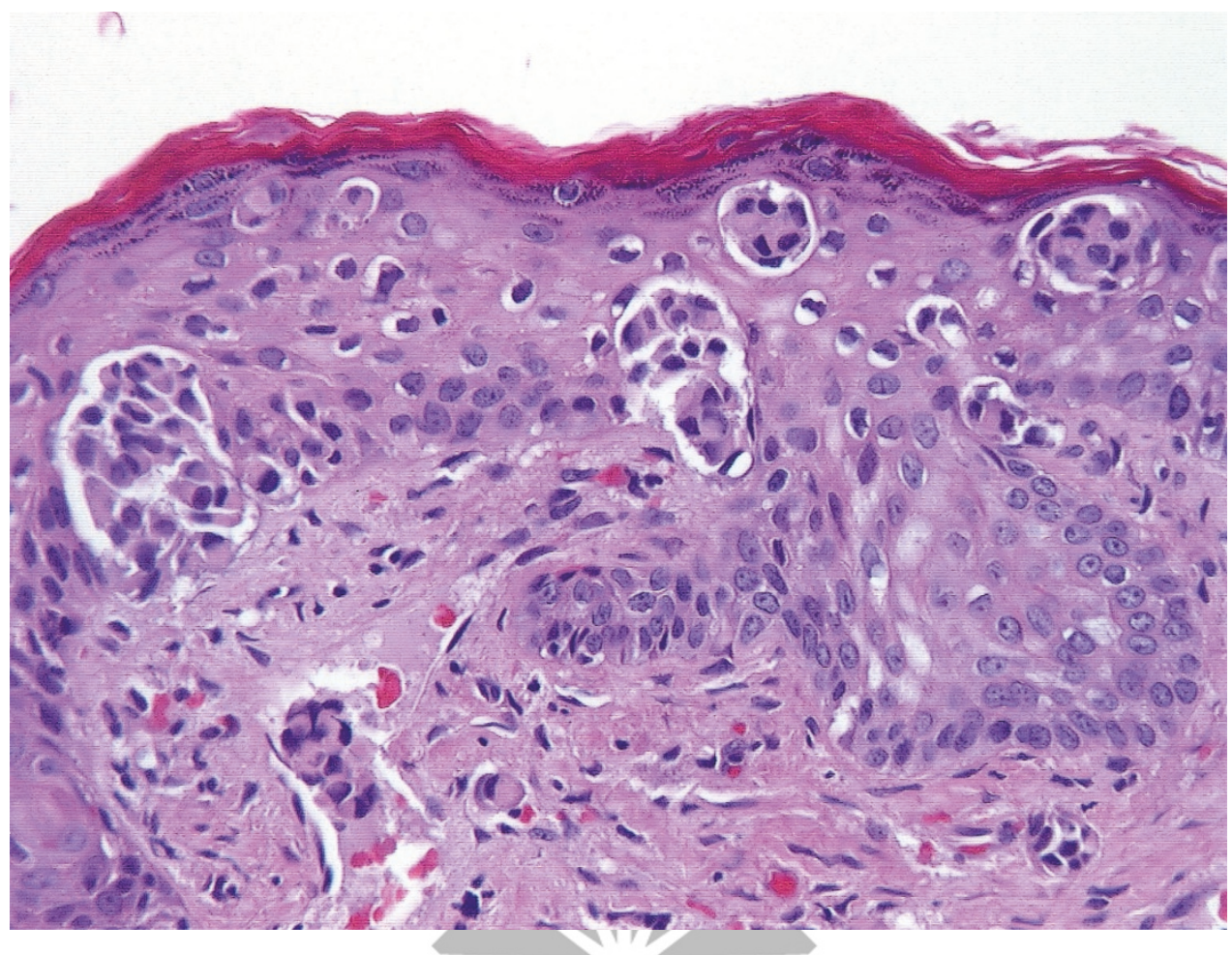

FIGURE 5. Pagetoid spread within metaplastic middle ear mucosa or external ear canal mucosa.

Table 5. Immunohistochemical Panel Results

\begin{tabular}{|c|c|c|}
\hline Antibody & $\begin{array}{c}\text { Positive } \\
\text { Reaction, } \mathrm{n} \\
\text { (\%) }\end{array}$ & $\begin{array}{c}\text { Intensity (\% of Cells } \\
\text { Reactive): no. of cases }\end{array}$ \\
\hline Cytokeratin & $43(89.6)$ & $\begin{array}{l}4+(>75 \%): 24 \\
4+(<25 \%): 10\end{array}$ \\
\hline Keratin 7 & $43(89.6)$ & $\begin{array}{l}4+(>75 \%): 5 \\
4+(<25 \%): 38\end{array}$ \\
\hline Keratin 20 & $3(6.4)$ & $1+-3+(>75 \%): 3$ \\
\hline CAM5.2 & $39(81.3)$ & $\begin{array}{c}2+(10-90 \%): 11 \\
3+(10-90 \%): 12 \\
4+(>75 \%): 14 \\
4+(<25 \%): 2\end{array}$ \\
\hline Chromogranin & $42(87.5)$ & $\begin{array}{c}1+(10-90 \%): 9 \\
2+(>75 \%): 13 \\
2+(<25 \%): 2 \\
3+(10-90 \%): 11 \\
4+(10-90 \%): 7\end{array}$ \\
\hline Synaptophysin & $15(31.3)$ & $\begin{array}{c}1+(10-90 \%): 6 \\
2+(50-90 \%): 6 \\
3+(>90 \%): 3\end{array}$ \\
\hline Serotonin & $12(25.0)$ & $\begin{array}{l}2+(10-75 \%): 3 \\
3+(<1-50 \%): 6 \\
4+(<1-90 \%): 3\end{array}$ \\
\hline Neuron-specific enolase & $24(50.0)$ & $\begin{array}{c}1+(50-75 \%): 4 \\
2+(<1->90 \%): 14 \\
3+(<25->90 \%): 6\end{array}$ \\
\hline Human pancreatic polypeptide & 45 (93.8) & $\begin{array}{c}2+(<10->90 \%): 6 \\
3+(<10->90 \%): 9 \\
4+(<10->90 \%): 30\end{array}$ \\
\hline $\begin{array}{l}\text { S-100 protein } \\
\text { Lesional cells } \\
\text { Stromal cells }\end{array}$ & $\begin{aligned} 23 & (48) \\
15 & (31.3) \\
8 & (18.8)\end{aligned}$ & $\begin{array}{c}1+-4+(<1->90 \%): 15 \\
2+-4+(>75 \%): 8\end{array}$ \\
\hline Vimentin & $48(100)$ & $4+(>90 \%): 48$ \\
\hline
\end{tabular}

HPP was at least focally positive in nearly all cases $(45 / 48,93.8 \%)$. Reactivity occurred in the same distribution as the other neuroendocrine markers and tended to be of moderate-to-strong intensity (Fig. 7). The three nonreactive cases displayed positive staining for NSE, chromogranin, synaptophysin, and/or serotonin.

${ }_{\mathrm{S}-100}$ was positive in the lesional cells in $15 \%$ of cases. Interestingly, strong reactivity was also noted within the stromal nuclei in $18 \%$ of the lesions. The significance of this finding is unclear but may indicate a stromal dendritic cell reaction to the invading tumor cells. Se

\section{Clinical Therapy and Patient Outcome}

All tumors were managed by surgery. The surgical procedures were most often excisional biopsy via a simple or modified mastoidectomy. Radical mastoidectomy was performed in cases where tumor encased the ossicles. Secondary reconstructive procedures were not documented. Radiation therapy or chemotherapy was not used for any of the cases reviewed.

Ten patients developed recurrent disease or have not been disease free. Two patients elected not to have a definitive or wide excision, and so over the course of 11.6 and 13.6 years, respectively, these patients have had multiple "biopsies" to remove the bulk of the tumor in each case, but have never had a definitive resection. If these two patients are excluded from further consideration, eight patients 


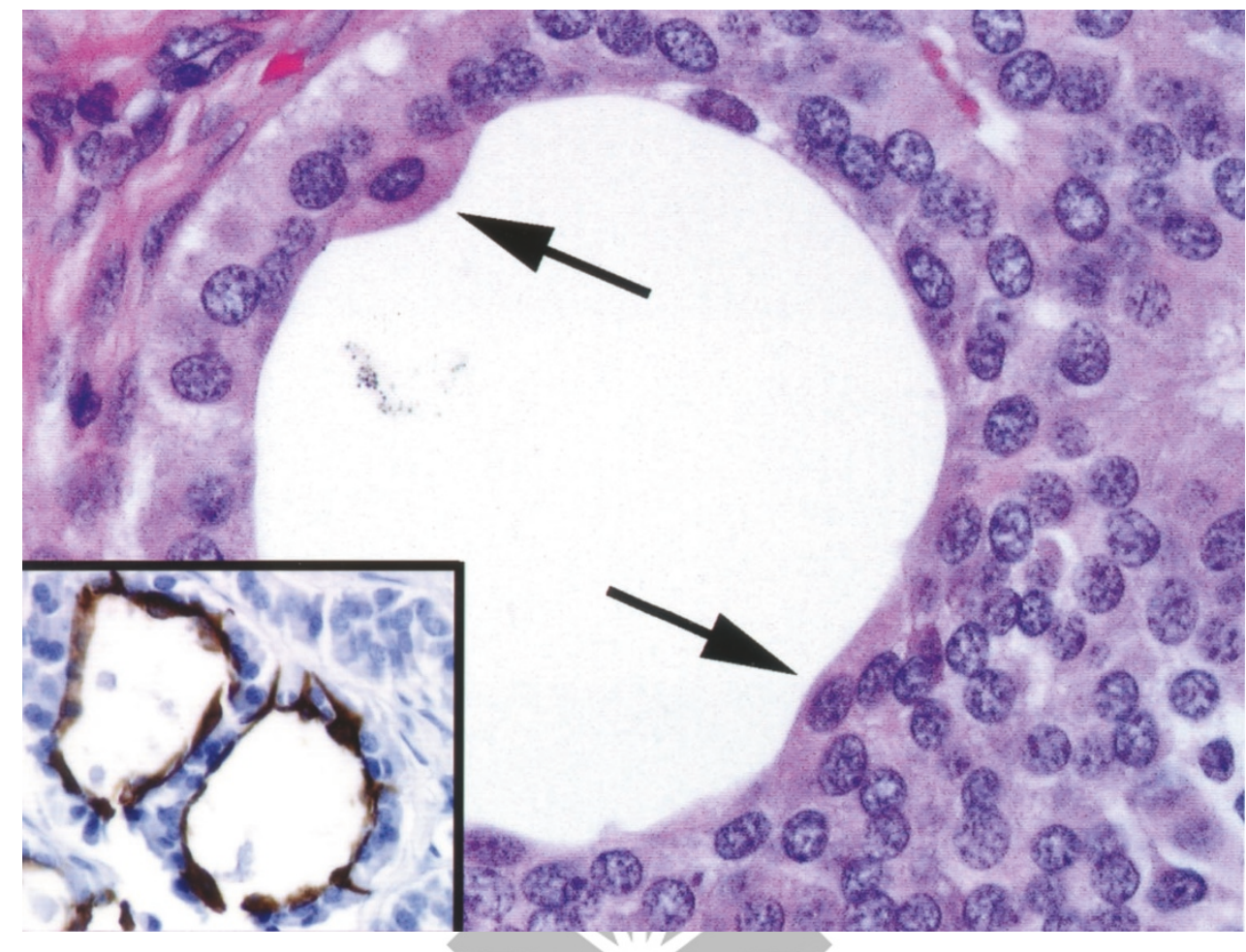

FIGURE 6. Glandular lumen with an inner flattened layer (arrows), surrounded by an outer cuboidal layer. Cytokeratin 7 immunohistochemistry (bottom left) highlights the inner epithelial layer.

developed recurrences. These eight patients in- tion of 14.0 months. The mean size of the tumor cluded six men and two women, aged 32-65 years, was $1.0 \mathrm{~cm}$, with seven tumors involving the left with an average age at presentation of 46.9 years. and one the right. Only one tumor extended into The symptoms were similar, with an average dura- the mastoid bone. Two tumors had moderate nu-

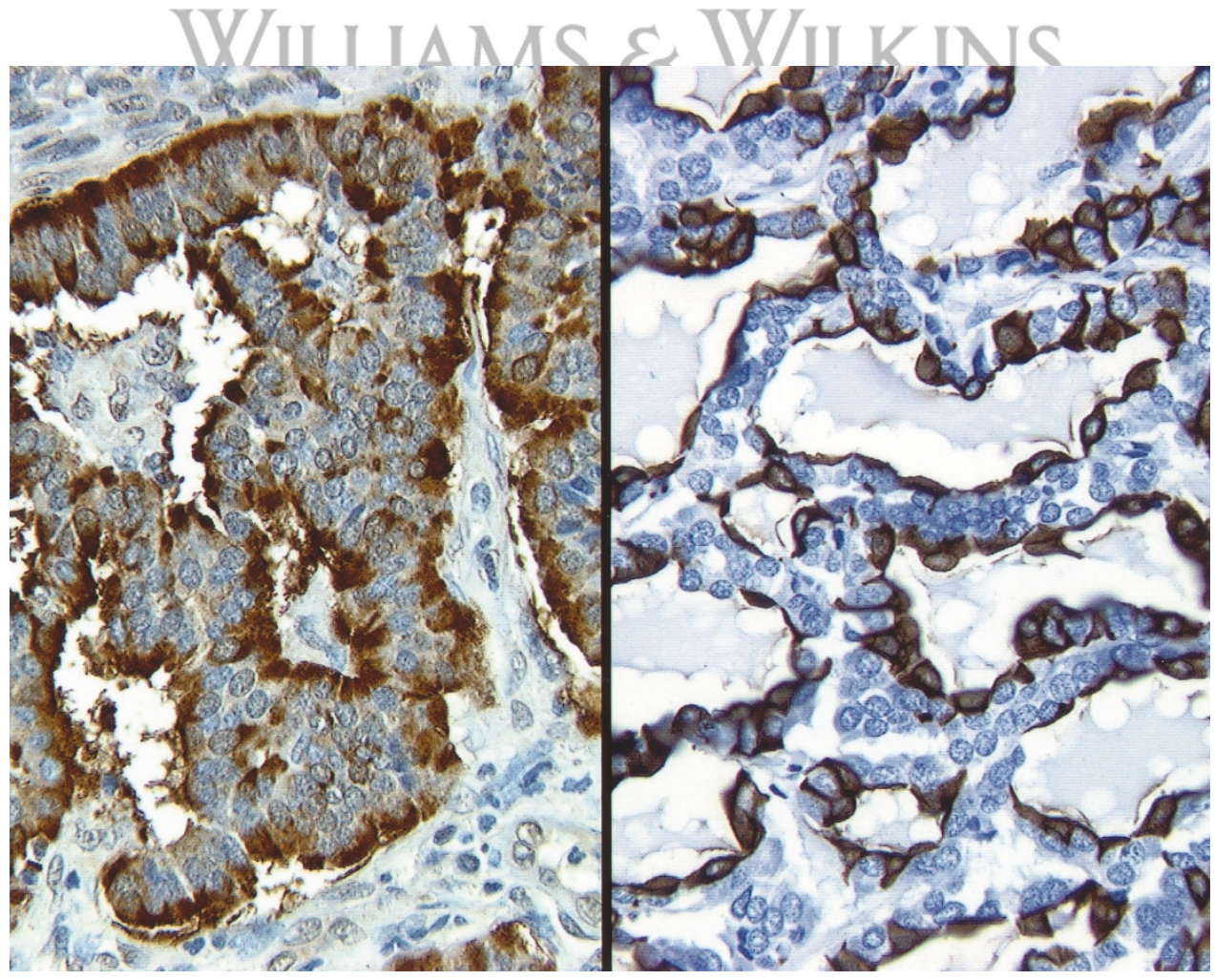

FIGURE 7. Human pancreatic polypeptide (left) and cytokeratin 7 (CK7; right) immunohistochemistry. Note the predominantly basilar staining pattern of the HPP and the inner, luminal pattern of the CK7. 
Table 6. Overall Patient Outcome (All Follow-up in Years)

\begin{tabular}{|c|c|c|c|c|}
\hline Group & All Cases ${ }^{a}$ & $\begin{array}{l}\text { Alive, } \\
\text { NED }\end{array}$ & $\begin{array}{l}\text { Alive, } \\
\text { D }\end{array}$ & $\begin{array}{c}\text { Dead, } \\
\text { NED }\end{array}$ \\
\hline All cases, $n$ (follow-up in y) & $45(15.7)$ & $37(16.9)$ & $2(12.6)$ & $6(8.7)$ \\
\hline Patients with extension, $n$ (follow-up in y) & $5(13.7)$ & $4(16.9)$ & $\mathrm{n} / \mathrm{a}$ & $1(1.0)$ \\
\hline Patients with recurrences, $n$ (follow-up in y) & $10(14.6)$ & $7(15.2)$ & $2(12.6)$ & $1(13.8)$ \\
\hline
\end{tabular}

${ }^{\text {a }}$ Follow-up was available in 45 of the 48 patients; NED, no evidence of disease; D, with disease; n/a, nonapplicable.

clear pleomorphism and two had high cellularity. All were managed by excisional biopsy ( $n=8$ patients). In all recurrences, the initial excision was conservative, leaving the ossicular chain intact. At the time of the recurrence, slightly more aggressive surgical excisions resulted in a clinical cure, especially when the involved ossicles were removed with the tumor.

There were no significant differences in morphology, immunohistochemistry, clinical presentation, or treatment outcomes in the group of patients analyzed. These findings lead to the conclusion that only one type of neoplasm was represented in our clinical series, which displayed numerous different architectural patterns but similar cytomorphology and immunohistochemical reactive patterns.

\section{DISCUSSION}

\section{Carcinoid Tumor versus Middle Ear Adenoma}

Benign neuroendocrine lesions of the middle ear have been described by many names, including carcinoid tumor (CT) (3), middle-ear adenoma (MEA) (2), adenomatous tumor of the middle ear ( 1 , 5), adenocarcinoid, and amphicrine tumor (6), with carcinoid tumor and middle-ear adenoma being the most accepted. There has been much debate in the literature as to the true nature of these neoplasms and whether they are similar, related but dissimilar, or totally separate entities. We feel that they represent the same tumor with different degrees of glandular and neuroendocrine differentiation.

Review articles of both MEAs and CTs have described a similar average age at presentation of approximately 37 years and a roughly equal gender distribution (2, 7-10). Clinical presentation has also been similar, with decreased hearing acuity, fullness, and tinnitus being the most frequently expressed symptoms. These findings are similar to those of this clinical series, although our average age (45 y) was slightly older.

At surgery, carcinoid tumors and middle-ear adenomas are both tightly adherent to the middle-ear cavity, commonly encasing the ossicular chain. Perforation of the tympanic membrane with extension into the external auditory canal was an occasional feature $(2,7,9,11)$. However, destruction of or invasion into the surrounding bone has only rarely been reported $(7,9,12)$, is not extensive in nature, and tended to bony erosion rather than destruction. Either tumor tended to be avascular, gritty, fibrous, or rubbery and appeared pale reddish-gray or yellow-tan (13).

Microscopically, middle-ear adenomas and carcinoid tumors have also been described as displaying similar, if not indistinguishable morphologies $(2,7-9,11)$. Architecturally, the neoplasms are arranged in glandular spaces, trabeculae, festoons, ribbon-like patterns, anastomosing cords, and solid sheets with variable cohesiveness. The glandular spaces commonly contained an amorphous secretory product that stained with mucicarmine, periodic acid-Schiff, and Alcian blue. The predominant architectural pattern tended to vary between tumors and even within the same tumor. In both neoplasms, the cells are described as cuboidal-tocolumnar, uniform in size, with eosinophilic, finely granular cytoplasm. The nuclei are round-to-oval with finely dispersed chromatin and may be centrally or eccentrically placed. A plasmacytoid appearance may be noted (14). Nucleoli are inconspicuous and mitoses are essentially absent. All these cytomorphologic and architectural features were observed within this clinical study.

Immunohistochemical staining for both MEAs and CTs has demonstrated similar results $(5,6,11$, 14-17). Cytokeratin cocktail and vimentin immunostaining have been uniformly positive. Neuroendocrine markers such as NSE, chromogranin, and synaptophysin have been uniformly present, although not necessarily all in the same tumor. Variable positivity for S-100, serotonin, glucagon, Leu-7, $\mathrm{ACTH}$, and somatostatin has also been noted. Human pancreatic polypeptide was uniformly positive within both tumors with only rare exception (two of our reported cases; 16). Although lysozyme immunoreactivity was considered essential for the diagnosis of MEA by one author (7), its absence has been noted by others $(5,11,14,16,17)$, and we did not perform this reaction in this clinical series.

The location and degree of positivity of the immunohistochemical staining varied with the architectural pattern. In small series and in this clinical study, CK was most strongly deposited in the cytoplasm of the glandular component and weakest within the solid component $(15,16)$. Neuroendocrine markers were most strongly identified in the trabecular, ribbon, and solid components while 
only weakly demonstrated in the glandular component $(16,17)$. Keratin 7 demonstrated a distinctly glandular specificity, especially for the inner lining cells of the glandular lumina, a finding not reported before now. These architecturally associated differences in immunohistochemical staining may have led different authors to favor either MEA or CT.

Although not performed for this study, middleear adenomas and carcinoid tumors have been extensively examined by the use of electron microscopy, and the results were similar $(5,6,11,15$, 17-19). Electron microscopy demonstrated two distinct cell types, so-called Type A and Type B cells. The Type A cells were noted within the apical aspect of the glandular lumina and rarely scattered within the solid areas. These cells exhibited a flattened apical pole and thin, elongated microvilli. Most significantly, these cells demonstrated exocrine activity with apically oriented 275-600 nm secretory granules composed of a flocculent dense core and an electron-lucent rim, representative of mucus granules. The granules were occasionally noted to fuse with the apical cellular membrane, thereby discharging their secretory product.

The Type B cells were noted in the basal aspect of the glandular structures and were the predominant cell within the solid, trabecular, and ribbon-like formations. They were most noteworthy for their basally located 110-350-nm cytoplasmic densecore neurosecretory granules that were surrounded by a thin halo and distinct limiting membrane. Furthermore, a transitional form exhibiting features of both Type A and Type B cells has also been described (11).

The immunohistochemical findings support the electron microscopic features $(5,6,11,14-18,20$, 21). The Type A cells found within the glandular lumens stained with keratins, especially keratin 7 . Cytokeratin staining was still present, although less intensely, within the Type B cells found within the solid, trabecular and other components. Conversely, the Type A cells were negative for neuroendocrine markers, whereas the Type B cells displayed intense immunoreactivity in keeping with their neuroendocrine nature.

The clinical (age, gender predilection), histologic, immunohistochemical, and electron microscopic features of these middle-ear neoplasms are similar to those of carcinoid tumors found within the lung and gastrointestinal tract (22-28). Arguments against the diagnosis of a carcinoid tumor within the middle ear have stemmed primarily from its lack of paraneoplastic syndromes (i.e., "carcinoid syndrome") and lack of documented metastasis to regional lymph nodes or distant sites. Paraneoplastic systemic alterations caused by the elaboration of hormonal products are a well-known feature of carcinoid tumors, especially the so-called "carcinoid syndrome" consisting of flushing, diarrhea, sweating, wheezing, and abdominal pain. However, lung carcinoid tumors only present with systemic alterations in less than 5\% of cases. When systemic changes are present, these lung tumors tend to be large $(>3.5 \mathrm{~cm} ; 28)$. The correlation of large tumor size to increased levels of hormone elaboration is logical, although these large tumors also have a greater frequency of liver metastasis that increases the chance of a paraneoplastic syndrome. With this in mind, the lack of systemic changes associated with those tumors of the middle ear may be more a function of their relatively small size (average, 0.8 $\mathrm{cm}$ in this study) rather than a lack of biologic potential. This is supported by their immunohistochemical staining for hormone products such as serotonin, ACTH, glucagon, HPP, and somatostatin. In fact there is a case of carcinoid tumor of the middle ear presenting with carcinoid syndrome (flushing, palpitation, dizziness, and diarrhea; 29). Unfortunately, no account was given as to the relative time frame between the disappearance of symptoms and the removal of the tumor, nor were the symptoms investigated by additional biochemical analysis because they were not initially thought to be related to the patient's complaints.

The metastatic rate for typical carcinoids of the lung is approximately $23 \%$ (28). Those that do metastasize tend to go to regional lymph nodes or the liver. The lack of documented metastasis of the tumors of the middle ear may be explained by the smaller size of the neoplasms in addition to the relative lack of vascularity of the middle-ear cavity when compared with the lung. Only a single case of metastasis to cervical lymph nodes has been documented (30). This tumor was initially treated with external beam radiation and developed multiple recurrences. The metastatic foci histologically showed significant pleomorphism and moderate mitotic activity, both of which were lacking in the previous biopsies of the middle-ear mass. Although attributed to the tumors' inherent biologic potential for metastasis, we feel that the history of treatment with radiation therapy and the significant change in cytomorphology more likely represented a radiation-induced malignant transformation.

\section{Etiology}

Carcinoid tumors of the lung are thought to originate from enterochromaffin cells (Kulchitsky cells), which are neuroendocrine cells normally present in the lung parenchyma. These cells are considered to originate from an endodermally derived pluripotential stem cell $(26,27)$. In common with the lung, the mucosa of the middle ear is also derived from the endoderm $(15,31,32)$. However, epithelial cells with neuroendocrine characteristics (such as the 
Kulchitsky cells of the lung) are not noted within the middle-ear cavity, either in healthy or inflamed states (5, 33-35). Therefore, an easily identifiable progenitor cell is lacking within the middle ear, although an undifferentiated, pluripotential endodermal stem cell may still be present within the surface mucosa of the middle ear that may give rise to carcinoid neoplasms similar to the cell of origin hypothesized for the lung (17).

Hyams and Michaels (2) were the first to hypothesize that middle-ear adenomas originated from the mucosal epithelium of the middle ear. This was due in part to the perception of tumor cells within the surface epithelium with invasion of the underlying stroma. We were unable to confirm this finding, either in this clinical series or in a review of the literature. In addition, critical analysis of the photomicrograph within the article used to document surface derivation (Fig. 5 on pg. 20) shows it to be more in keeping with a ceruminal adenoma with a thick, apparently bilayered epithelium forming ductal structures with many papillations and decapitation apocrine secretions. This is in contrast to the thin, non-papillated glandular pattern found in the rest of their cases (specifically Fig. 1) and in this clinical series. Mills and Fechner (7) are attributed with observing actual derivation from the surface mucosa. However, not only is this feature not documented by a photomicrograph, but it is also described as tumor cells "replacing" the overlying epithelium as opposed to originating from it. This is similar to our observation of pagetoid-like spread noted within two of the neoplasms in this series.

The lack of evidence for surface epithelial derivation leads to the consideration of a stromal precursor. The stroma of the middle ear is derived from mesoderm and the neural crest $(31,32,36)$. The neural crest gives rise to parts of the ossicular chain and the three primary paraganglia (tympanic glomus, jugular glomus, and satellite glomus of Curveilhier-Arnold) $(37,38)$ The glomus cells normally display neuroendocrine characteristics with neuroendocrine dense core granules and positive immunohistochemical staining for NSE, chromogranin, and/or synaptophysin. Epithelial or exocrine characteristics are not normal features of these cells. However, rare paragangliomas have been noted to show CK positivity indicative of epithelial expression or perhaps even transformation $(6,39,40)$. Therefore, it is plausible that a neuroendocrine neoplasm of the middle ear may originate from a neural crest-derived stem cell.

In either event, a metaplastic alteration would be required within resident cells of the middle ear to account for the dual population noted within these tumors. Either uncommitted epithelial (endodermal) cells would develop neuroendocrine features or uncommitted neural crest cells would acquire exocrine and epithelial characteristics. Despite the lack of evidence for a surface mucosal origin, their close histologic and clinical relationship to carcinoid tumors of the lung favors an origin from an uncommitted endodermal stem cell. It is plausible that endodermally derived stem cells may have become sequestered within the stroma of the middle ear during embryogenesis, thereby explaining the predominant stromal location of these tumors.

\section{Benign or Malignant?}

Most authors consider typical carcinoid tumors of the lung to behave as low-grade malignant tumors. This is supported by their low, but still significant metastatic potential $(<23 \% ; 28)$. An alteration in nomenclature to "well-differentiated neuroendocrine tumor" has been proposed to better define the histologic, morphologic, and clinical characteristics of typical carcinoid tumors (25). Although not yet adopted by the World Health Organization, the nomenclature of well-differentiated neuroendocrine tumor, well-differentiated neuroendocrine carcinoma, and poorly differentiated neuroendocrine carcinoma are logical replacements for typical carcinoid, atypical carcinoid, and small cell carcinoma of the lung, respectively.

Carcinoid tumors of the middle ear, unlike their relatives in the lung, have not shown metastatic potential. Whether because of a difference in biologic potential between the middle ear neoplasms and their supposed pulmonary counterparts or because of the anatomic constraints of the middle ear and its relative lack of vascularity, these tumors clinically behave as benign entities. Bone invasion and destruction is highly uncommon with these lesions and, when present, is more in keeping with low-grade adenocarcinomas of endolymphatic sac origin (endolymphatic sac tumors; 31 ) or metastatic neoplasms to the middle ear. The recurrence rate for these neuroendocrine neoplasms of the middle ear is also quite small (see below). When they do recur, it is usually related to regrowth of an incompletely excised tumor as opposed to an inherent biologic aggressiveness. Case reports have been presented of unilateral facial paralysis associated with this tumor $(17,41-43)$. Indeed, three of the cases in this clinical series also displayed nerverelated symptoms. Nevertheless, facial paralysis does not seem to portend an adverse clinical outcome as all patients in this series, and those within the literature with adequate follow-up had these symptoms resolve after complete tumor removal. These findings suggest mass-related compression of the facial nerve rather than direct neural invasion. In summary, if one defines "malignancy" by clinical aggressiveness and potential for metastatic spread, then the tumors of the middle ear should be regarded as benign. 


\section{Nomenclature}

Typical carcinoid tumor, by definition, is a lowgrade malignant neoplasm. Although the tumors within the middle ear are histologically (by light and electron microscopy) and immunohistochemically similar to carcinoid tumors, their benign clinical nature precludes including them within this group. Although the neuroendocrine features of this tumor are not adequately conveyed by middleear adenoma, it is a correct description of the benign nature of the tumor and its location. Neuroendocrine adenoma of the middle ear may be an excellent alternative as it suggests a correct morphologic and behavioral moniker of this rare neoplasm.

\section{Treatment and Prognosis}

Complete surgical removal of the neoplasm, to include encased ossicles (if present), should be the treatment of choice $(7,11,35,44)$. When the ossicular chain is involved but not removed, recurrence (regrowth) of the lesion is much more likely. With this said, two of the patients within our study were being treated with repeated debulking procedures to preserve the ossicular chain and thereby maintain hearing. This practice, although not supported nor discouraged by the authors, would require excellent continued long term clinical follow-up and patient compliance. In addition, the biologic potential for true malignant transformation of longstanding tumors has not been studied, although must be exceedingly uncommon given the scarcity of/reported cases and no transformations identified in this large clinical study.

Radiation therapy is not required for these tumors (45). In fact, secondary malignant transformation of these tumors is a possible outcome if our hypothesis for the one reported case of metastatic spread is correct (30).

This clinical study uncovered an approximate $18 \%$ recurrence rate (not including those two patients who elected to forgo complete surgical excision). All recurrences developed when the ossicular chain was not excised during initial surgery, thereby potentially leaving the patient with residual tumor. Subsequent surgical procedures to thoroughly remove all involved structures resulted in a good clinical outcome. Although we did not document recurrences in patients who had initial complete removal of the tumor, other investigators have reported recurrence in isolated patients $(7,45)$.

\section{SUMMARY}

Carcinoid tumor and middle-ear adenoma are two well-documented, albeit rare, neoplasms of the middle ear. Despite considerable debate over the perceived similarities and differences between these two tumors, we believe that they actually represent the same tumor. We draw this conclusion based on similarities in clinical, histologic (by light and electron microscopy), and immunohistochemical results, both within our study and in the review of the literature. Although akin to that of typical carcinoid tumors of the lung, the middle-ear neoplasms' clinical behavior merits a benign designation. We favor middle-ear adenoma as it correctly describes the benign clinical nature of this neoplasm, although neuroendocrine adenoma of the middle ear may be an even better designation. Treatment consists of complete surgical excision with removal of the ossicular chain, if involved. Recurrence is rare with initial complete excision.

Acknowledgment: The authors thank Dr. Dennis Heffner for his critical analysis of the manuscript, insight, and support.

\section{REFERENCES}

1. Derlacki EL, Barney BP. Adenomatous tumors of the middle ear and mastoid. Laryngoscope 1976;86:1123-35.

2. Hyams VJ, Michaels L. Benign adenomatous neoplasm (adenoma) of the middle ear. Clin Otolaryngol 1976;1:17-26.

3. Murphy GF, Pilch BZ, Dickersin GR, Goodman ML, Nadol JBJ. Carcinoid tumor of the middle ear. Am J Clin Pathol 1980;73:816-23.

4. Hsu SM. Immunohistochemistry. N Engl J Med 1990;184: 357-63.

5. McNutt MA, Bolen JW. Adenomatous tumor of the middle ear. An ultrastructural and immunocytochemical study. Am J Clin Pathol 1985;84:541-7.

6. Ketabchi S, Massi D, Franchi A, Vannucchi P, Santucci M. Middle ear adenoma is an amphicrine tumor: why call it adenoma? Ultrastruct Pathol 2001;25:73-8.

7. Mills SE, Fechner RE. Middle ear adenoma. A cytologically uniform neoplasm displaying a variety of architectural patterns. Am J Surg Pathol 1984;8:677-85.

8. Riddell DA, Le Boldus GM, Joseph MG, Hearn SA. Carcinoid tumour of the middle ear: case report and review of the literature. J Otolaryngol 1994;23:276-80.

9. Hosoda S, Tateno H, Inoue HK, Isojima G, Kondo S, Konishi T. Carcinoid tumor of the middle ear containing serotonin and multiple peptide hormones. A case report and review of the pathology literature. Acta Pathol Jpn 1992;42:614-20.

10. Himi T, Saitoh H, Ohguro S, Yoshioka I, Kataura A. Carcinoid tumor of the middle ear and mastoid. Auris Nasus Larynx 1995;22:128-33.

11. Manni JJ, Faverly DR, Van Haelst UJ. Primary carcinoid tumors of the middle ear. Report on four cases and a review of the literature. Arch Otolaryngol Head Neck Surg 1992;118: 1341-7.

12. Hardingham M. Adenoma of the middle ear. Arch Otolaryngol Head Neck Surg 1995;121:342-4.

13. Woods RH, Moses B, Lumpkin S, Pearlman S. Middle ear adenoma: report of two cases. Otolaryngol Head Neck Surg 1993;108:754-9.

14. Ribe A, Fernandez PL, Ostertarg H, Claros P, Bombi JA, Palacin A, et al. Middle-ear adenoma (MEA): a report of two 
cases, one with predominant "plasmacytoid" features. Histopathology 1997;30:359-64.

15. Stanley MW, Horwitz CA, Levinson RM, Sibley RK. Carcinoid tumors of the middle ear. Am J Clin Pathol 1987;87:592-600.

16. Paraskevakou H, Lazaris AC, Kandiloros DC, Papadimitriou K, Adamopoulos G, Davaris PS. Middle ear adenomatous tumor with a predominant neuroendocrine component. Pathology 1999;31:284-7.

17. Wassef M, Kanavaros P, Polivka M, Nemeth J, Monteil JP, Frachet B, et al. Middle ear adenoma. A tumor displaying mucinous and neuroendocrine differentiation. Am J Surg Pathol 1989;13:838-47.

18. Riches WG, Johnston WH. Primary adenomatous neoplasms of the middle ear: light and electron microscopic features of a group distinct from the ceruminomas. Am J Clin Pathol 1982;77:153-61.

19. Davies JE, Semeraro D, Knight LC, Griffiths GJ. Middle ear neoplasms showing adenomatous and neuroendocrine components. J Laryngol Otol 1989;103:404-7.

20. Hale RJ, McMahon RF, Whittaker JS. Middle ear adenoma: tumour of mixed mucinous and neuroendocrine differentiation. J Clin Pathol 1991;44:652-4.

21. Kochilas X, Birchall JP, Sloan P. Light and electron microscopic studies of a case of middle ear adenoma. Laryngoscope 1984;94:1482-4.

22. Rosai J. Carcinoid. In: Rosai J, editor. Ackerman's surgical pathology. 8th ed. Mosby; 1996. p. 392-8.

23. Colby TV, Koss MN, Travis WD. Carcinoid and other neuroendocrine tumors. In: Rosai J, Sobin LH, editors. Pathology of the lower respiratory tract. 3rd ed. Washington, DC: Armed Forces Institute of Pathology; 1995. p. 287-309.

24. Ghadially FN. Ultrastructural pathology of the cell matrix, 4th Edition. Boston: Butterworth-Heineman, 1997.

25. Kulke MH, Mayer RJ. Carcinoid tumors. N Engl J Med 1999; 340:858-68.

26. Warburton D, Wuenschell C, Flores-Delgado G, Anderson K. Commitment and differentiation of lung cell lineages. Biochem Cell Biol 1998;76:971-95.

27. Brambilla E, Lantuejoul S, Sturm N.-Divergent differentiation in neuroendocrine lung tumors. Semin Diagn Pathol 2000; $17: 138-48$.

28. Soga J, Yakuwa Y. Bronchopulmonary carcinoids. Ann Thorac Cardiovasc Surg 1999;5:211-9.

29. Latif MA, Madders DJ, Barton RP, Shaw PA. Carcinoid tumour of the middle ear associated with systemic symptoms. J Laryngol Otol 1987;101:480-6.
30. Mooney EE, Dodd LG, Oury TD, Burchette JL, Layfield LJ, Scher RL. Middle ear carcinoid: an indolent tumor with metastatic potential. Head Neck 1999;21:72-7.

31. Kempermann G, Neumann HP, Volk B. Endolymphatic sac tumours. Histopathology 1998;33:2-10.

32. Mallo M. Formation of the middle ear: recent progress on the development and molecular mechanisms. Dev Biol 2001; 231;410-9.

33. Gunduz M, Yamanaka N, Saito T, Kuki K, Yokoyama M, Nakamine H. Middle ear adenoma with neuroendocrine differentiation. Auris Nasus Larynx 2000;27:73-6.

34. Batsakis JG. Adenomatous tumors of the middle ear. Ann Otol Rhinol Laryngol 1989;98:749-52.

35. Arnold B, Zietz C, Muller-Hocker J, Wustrow TP. Adenoma of the middle ear mucosa. Eur Arch Otorhinolaryngol 1996;253:65-8.

36. Bold EL, Wanamaker JR, Hughes GB, Rhee CK, Sebek BA, Kinney SE. Adenomatous lesions of the temporal bone immunohistochemical analysis and theories of histogenesis. Am J Otol 1995;16:146-52.

37. Amble FR, Harner SG, Weiland LH, McDonald TJ, Facer GW. Middle ear adenoma and adenocarcinoma. Otolaryngol Head Neck Surg 1993;109:871-6.

38. Mafee MF, Raofi B, Kumar A, Muscato C. Glomus facial, glomus jugulare, glomus tympanicum, glomus vagale, carotid body tumors, and simulating lesions. Radiol Clin North Am 2000;38:1059-76.

39. Labrousse F, Leonard GL, Petit B. Cytokeratins expression in paragangliomas of the cauda equina. Clin Otolaryngol 1999; 18:208-13.

40. Chetty R, Pillay P, Jaichand V. Cytokeratin expression in adrenal pheochromocytomas and extra-adrenal paragangliomas. J Clin Pathol 1998;51:477-8.

41. Zahtz GD, Zielinski B, Abramson AL. Benign adenoma of the middle ear cavity causing facial paralysis. Otolaryngol Head Neck Surg 1981;89:624-7.

42. Jahrsdoerfer RA, Fechner RE, Moon CNJ, Selman JW, Powell JB II. Adenoma of the middle ear. Laryngoscope 1983;93:1041-4.

43. Hagan WE, Leonard GL, Ichinose H, Cox RH. Primary monomorphic adenoma of the middle ear. Laryngoscope 1980;90: 1962-72.

44. Noel FL, Benecke JEJ, Carberry JN, House JW, Patterson M. Adenomas of the mastoid and middle ear. Otolaryngol Head Neck Surg 1991;104:133-4.

45. Krouse JH, Nadol JBJ, Goodman ML. Carcinoid tumors of the middle ear. Annu Otol Rhinol Laryngol 1990;99:547-52. 